\title{
A statistical analysis of the detection limits of fast photometry
}

\author{
D. L. Mary ${ }^{1,2}$ \\ 1 Aryabhatta Research Institute of Observational Sciences (ARIES), Manora Peak, NainiTal-263129, India \\ 2 Astronomisches Rechen Institut, am Zentrum für Astronomie, Moenchoffstrasse 12-14, 69120 Heidelberg, Germany \\ e-mail: dmary@ari.uni-heidelberg.de
}

Received 26 October 2005 / Accepted 2 February 2006

ABSTRACT

\begin{abstract}
This work investigates the statistical limits for the detection of stellar variability using ground based fast photometry. We show that when sky transparency variations are very low or have been efficiently removed from the raw light curve, the overall noise is of a Mixed Poisson (MP) nature (photon noise mixed by scintillation). As a consequence, three regimes appear for the detection of photometric variations depending on the star's brightness (scintillation, scintillation and photon noise, photon noise and sky background). The proposed analysis is mainly applied to the Indian sites of Manora Peak (existing $104 \mathrm{~cm}$ telescope) and Devasthal (future $1 \mathrm{~m}$ automated telescope, and $3 \mathrm{~m}$ telescope project). As shown by some examples, it can be applied to any site with the corresponding parameters. For $1 \mathrm{~m}$ class telescopes at an altitude of about $2000 \mathrm{~m}$, the frontier magnitudes between the different detection regimes are about $10 \mathrm{mag}$ and $15 \mathrm{mag}$. By analysing the corresponding statistics of the MP noise periodogram, the minimum amplitude variation that one can detect with a given confidence level is evaluated for each observational setting. For example, with a $3 \mathrm{~m}$ telescope at about $2500 \mathrm{~m}, \approx 120 \mu \mathrm{mag}$ variations would be detected in $2 \mathrm{~h}$ with a $99 \%$ confidence level for stars brighter than magnitude 12 . For a star of 15 th magnitude, $\approx 400 \mu \mathrm{mag}$ oscillations would still be detected at that level. These detection limits are discussed in the light of observations obtained in Manora peak, and compared to results obtained at different astronomical sites.
\end{abstract}

Key words. techniques: photometric - stars: variables: $\delta$ Sct - stars: chemically peculiar

\section{Introduction}

The Combined General Catalogue of Variable Stars (GCVS, Kholopov et al. 1998) reports the variability characteristics of numerous classes of variable stars. The luminosity variations exhibited by these stars depend on their mass, structure, binarity, evolutionary status and chemical composition. Their variations may be periodic or quasi-periodic (pulsating stars, rotating variables, eclipsing binaries), or aperiodic (eruptive variables, optical reprocessing in X-ray binaries). Many variables have short timescales and small amplitude photometric variations. This paper proposes a statistical evaluation of the ability of groundbased photometry to detect rapid stellar variations with a high precision. The typical time scale of these oscillations ranges from minutes to hours. Because we are interested in detecting short timescale phenomena, the time sampling of the light curves is typically a few tens of seconds. The technique is called fast photometry. High precision photometry is important because the higher the precision, the stronger the constraint on the physical parameters. Indeed, some stars (e.g. pulsating roAp and some $\delta$ Scuti stars) must be observed with high photometric precision because the amplitude of their oscillations is low (of the order of a few mmag or even less). Many stars which were believed to be of constant luminosity have been discovered to be variable with high time resolution and high-precision photometry. Such discoveries open new windows for astrophysical investigation, in particular for the application of asteroseismologic tools.

The search for new variables is an active research field at the Aryabhatta Research Institute of Observational Sciences (ARIES), NainiTal, India. A collaborative program searching for milli magnitude pulsations in chemically peculiar (CP) stars in the northern hemisphere was initiated in 1997 between NainiTal, India, and Cape Town, South Africa (Martinez 2001; Joshi 2003, 2004; Girish 2004). Pulsating stars form an extremely rich subclass of variable stars. By analysing the pulsation patterns exhibited in the light curves, asteroseismology allows one to infer physical parameters for the stars (internal structure, effective temperature, magnetic field, gravity, mass, inclination angle ..., see e.g. Christensen-Dalsgaard 2003). The pulsations searched for in the NainiTal-Cape survey range from about 6 to $21 \mathrm{~min}$ in the case of rapidly oscillating A peculiar stars (roAp stars, Kurz 1985; Martinez 1993), and from $18 \mathrm{~min}$ to about $8 \mathrm{~h}$ for Delta Scuti-like variables (Breger 2000). Apart from CP stars, pulsating White Dwarfs are monitored at the ARIES in the WET (Whole Earth Telescope, Nather et al. 1990) framework (Reed et al. 2000), and a program of optical following of X-ray binaries has been started recently. The fast photometric observations are currently performed at Manora Peak (104 cm telescope, altitude $h=1951 \mathrm{~m}$ ), at the ARIES site. Similar measurements will be made by the end of 2006 at Devasthal $(\approx 50 \mathrm{~km}$ away from NainiTal, $h=2420 \mathrm{~m}$ ) where a $1 \mathrm{~m}$ fully automated telescope is being built. The ARIES has also the project to build a $3 \mathrm{~m}$ telescope at Devasthal.

The detection limits of stellar variations are known to depend on many parameters. Firstly, on the site: altitude, atmosphere (scintillation, sky transparency variations, extinction in the considered band, light pollution, etc.); secondly, on the telescope (telescope's aperture, reflectivity of primary and secondary mirrors); thirdly, on the position of the target star w.r.t. zenith (airmass); fourthly, on the observation's wavelength and on the filter bandwidth and finally on some physical constants. Indeed, the 
detector's performance also is important. It is well established however that for state-of-the-art photometric instruments, the stochastic light fluctuations caused by the atmosphere constitute the major limitation in photometry of relatively bright stars (see e.g. Martinez 1993; and Sagar \& Mary 2005, for comments on the instrumental noise of the 3-channel photometer used at the ARIES for the roAp star survey). Photon noise and sky background become non negligible for faint stars (more than about 11th mag for $1 \mathrm{~m}$ telescopes); the instrumental noise starts affecting the detection performance for even fainter stars. For this reason and because we do not want the following analysis to depend on a particular technology, the noise of the detector will not be accounted for in this analysis.

It has been known for a long time how one or a few particular factors above affect the detection (Brown \& Gilliland 1994; Martinez \& Kurtz 1994; Sagar \& Mary 2005). The characterisation of the overall noise in a global statistical setting has however not been carried out yet. In this framework, the aims of this work are twofold. Firstly, we are interested in modeling analytically the contributions of the noise sources in fast photometric observations, for any given star's magnitude and position in the sky, and for any given observational setting (site \& telescope). Secondly, we wish to derive the detection limits imposed by the overall noise. Both the cases of periodic and non periodic stellar variations are investigated. The results are applied to the sites of Manora Peak ( $1 \mathrm{~m}$ telescope), to the Devasthal site (1 and $3 \mathrm{~m}$ telescopes) and to other astronomical sites (Sect. 6). Also, for real data, we shall focus on the pulsating roAp and $\delta$ Scuti stars, but the proposed model is useful to assess the detectability of any variable stars with similar variability timescales.

The paper is organised as follows. The second section evaluates the number of photons to be detected and investigates the statistics of the noise photons. In the third section we quantify the contributions of signal and noise in the light curves, and the Signal-to-Noise Ratio (SNR) in the temporal domain. The fourth section evaluates the influence of the noise in the Fourier amplitude spectrum, and the $S N R$ in the spectrum. In the fifth section we evaluate the confidence level corresponding to a given $S N R$, and we derive analytically the minimal amplitude oscillation that can be detected with a given confidence level for a given astronomical site and telescope. The validity of this model is assessed in Sect. 6. The last section summarises the main results.

\section{Photons from stellar oscillations and photons from noise}

\subsection{Photons from signal}

In the following, the subscripts $*$ and sky correspond respectively to the star and to the sky background. (The sky background is the light coming from the sky, which is never perfectly dark. This light is indeed always detected in addition to that of the star.) The notations $F, f$ and $n$ denote respectively fluxes, magnitudes and numbers of photons. It is customary to use (milli)magnitudes [(m)mag, dimensionless] instead of fluxes $\left[\mathrm{erg} / \mathrm{cm}^{2} / \mathrm{s} / \AA\right]$. Evaluating the quantity of light by the number of photons is also useful because photons rather than fluxes are actually detected. We shall therefore use these three quantities equivalently. The relationship of magnitude to flux is $f=-2.5 \log _{10}\left[F / F_{*}(0)\right]$, where $F_{*}(0)$ is the reference flux at magnitude zero. The relationship of photons $n$ to flux $F$ is $n=F \Delta \lambda S\left(\frac{h c}{\lambda}\right)^{-1}$, where $\Delta \lambda, S, h, c$ and $\lambda$ are respectively the filter bandwidth $[\AA]$, the surface area of the telescope's aperture $\left[\mathrm{cm}^{2}\right]$, the Planck's constant [erg s], the speed of light $[\AA / \mathrm{s}]$ and the wavelength of light $[\AA ̊]$. The quantity $\frac{h c}{\lambda}$ is the energy of one photon [erg].

In the case where the stellar light variation is not periodic, it will take the form of a random light curve. For the purpose of making a model, we shall quantify this stellar variation by a root mean square (rms) variation in flux $\Delta F$ (or $\Delta f$ mag, or $\Delta n$ photons) with respect to the average stellar flux. In the case where the variation is periodic, the corresponding rms deviation in flux will be denoted by $\Delta F$ as well. We focus on the periodic case (pulsating stars typically) because we will examine the Fourier spectra later on (Sect. 4). The results of the present Section, which regards light curves, are valid in both the periodic and aperiodic cases: $\Delta F$ has just to be given the corresponding definition.

Let us assume that a target star of average magnitude $f_{*}$ and average flux $F_{*}$ oscillates with an amplitude (half peak to peak variation) of a few mmag $\Delta f_{\max }$. Without loss of generality, the oscillation can be assumed to be zero mean and sinusoidal This model is useful for both small and large stellar luminosity variations (see e.g. Poretti 2002; Christensen-Dalsgaard 2003; Mary 2005). Then the amplitude variation in mag with respect to the average stellar level $f_{*}$ is $\Delta f_{\max }=\sqrt{2} \Delta f$, or, in photons, $\sqrt{2} \Delta n$. With the definitions above, the rms deviation in magnitude $\Delta f$ corresponding to the stellar variation with respect to $f_{*}$ can easily be related to the rms deviation in photons $\Delta n$ by

$\Delta f=2.5 \log _{10}\left(1+\frac{\Delta n}{n_{*}}\right)$.

Using an analysis similar to that of Mayya (1991), Eq. (6), the corresponding number of photons (rms) falling on the detector during $T_{\text {int }}$ seconds is

$$
\begin{aligned}
\Delta n & =\Delta F \Delta \lambda S \in \eta 10^{\{-0.4 x(\lambda) \sec Z\}} T_{\text {int }}\left(\frac{h c}{\lambda}\right)^{-1} \\
& =\Delta F \alpha\left(\frac{h c}{\lambda}\right)^{-1},
\end{aligned}
$$

where $\epsilon, \eta, x(\lambda)$ and sec $\mathrm{Z}$ are respectively the reflectivity of the mirrors (dimensionless) and also the transmission of a Fabry lens (if present in the photometer under consideration), the quantum efficiency of the detector (dimensionless), the extinction in the considered band [mag] and the airmass. In Eq. (2), the coefficient $\alpha$ contains the parameters of the considered observational setting (site \& telescope).

The results below pertain to the Johnson $B$ band $(\lambda=$ $4380 \AA$ ) because pulsations of roAp stars are more prominent in this band. A filter of bandwidth $940 \AA$ is assumed. The values of the atmospheric parameters for the Indian sites come from extensive site measurements published by Sagar et al. (2000) and Kumar et al. (2000). In particular, the average values for the extinction in the blue at Manora Peak and Devasthal are 0.26 and $0.22 \mathrm{mag} /$ airmass respectively. The sky brightness $f_{\text {sky }}$ is $22.2 \mathrm{mag}$ in $B$ for both sites. Using these data, the average number of photons $n_{*}$ corresponding to $F_{*}$, and the number of photons $n_{\text {sky }}$ corresponding to the sky background can be derived in the same manner as in Eq. (2). A value of 15 arcsec for the aperture diameter is used to calculate the sky background. With a 30 arcsec aperture, we obtain very similar results. The only noticeable changes regard quite faint stars $(\approx 14$ th mag or more).

\subsection{Mixed-Poisson nature of the noise}

Now, because of two major random noise effects (scintillation and photon noise), the actual number of detected photons is random, even for a non variable star with constant flux $F_{*}$. This 
noise creates a scatter in the data points of the light curve. The noise scatter corresponds to a rms number of photons $\Delta n_{\text {noise }}$. This unavoidable scatter should be compared to $\Delta n$ of Eq. (2) to evaluate how much it perturbs the visibility of the stellar signal. For a given combination of site and telescope, the joint effects of scintillation and photon noise can be described and evaluated as follows.

Firstly, random changes in temperature occur in the (mainly upper) atmosphere, which in turn generate random fluctuations of the air's refractive index. Consequently, any deterministic flux propagating through these turbulent layers becomes random. The effects of these fluctuations are to randomly defocus the star, creating scintillation (see the seminal works of Young 1967; and Dravins 1997a,b, 1998). As such, scintillation involves the curvature (second order derivative) of the wavefront. The wavefront's phase randomness corresponding to seeing variations (related to the first order derivative) does not perturb photometric measurements if the photometric aperture is large enough.

The rms of the scintillation level, expressed in number of photons $\Delta n_{\mathrm{sc}}$, can be estimated from Dravins (1997a), Eq. (10). We obtain

$$
\begin{aligned}
\Delta n_{\mathrm{sc}} & =0.09 D^{-\frac{2}{3}}(\sec Z)^{1.75} \mathrm{e}^{-\frac{h}{h_{0}}}\left(2 T_{\mathrm{int}}\right)^{-\frac{1}{2}} n_{*} \\
& =\beta n_{*} .
\end{aligned}
$$

In the above equation, $D$ is the telescope diameter (in $\mathrm{cm}$ ), $h$ the altitude (1951 m for Manora Peak, $2420 \mathrm{~m}$ for Devasthal). The quantity $h_{0}$ corresponds to the upper value below which the model is considered as valid $(8000 \mathrm{~m})$. A typical integration time in fast photometry is $T_{\text {int }}=10 \mathrm{~s}$. All the results below are established for this value. In Eq. (3), the coefficient $\beta$ contains the parameters of the observational setting.

Secondly, the detection of the photons is random because of the quantum nature of light. In the absence of an atmosphere, the statistics of the detected photons for a non variable star with constant flux $F_{*}$ would be Poissonian, with mean and variance $n_{*}+n_{\text {sky }}$.

Accounting now for photon and scintillation noise together, the number of detected photons is doubly stochastic: the process is Poisson (because of detection noise) with a mean which is itself stochastic (because of scintillation). Let us denote the probability of detecting $n_{\text {tot }}$ photons given a flux $F$ by $P\left(n_{\text {tot }} \mid F\right)$, and by $p(F)$ the flux distribution caused by scintillation. From Eq. (2), the expected number of detected photons corresponding to a given flux $F$ is $\alpha \frac{\lambda}{h c} F$. But because $F$ is random, we have to account for all the values of $F$ to get the actual distribution of the number of detected photons $n_{\text {tot }}$. The probability of detecting $n_{\text {tot }}$ photons is then

$$
\begin{aligned}
P\left(n_{\mathrm{tot}}\right) & =\int_{0}^{+\infty} P\left(n_{\mathrm{tot}} \mid F\right) p(F) \mathrm{d} F \\
& =\int_{0}^{+\infty}\left(\frac{\alpha \lambda}{h c} F\right)^{n_{\mathrm{tot}}} \mathrm{e}^{-\frac{\alpha \lambda}{h c} F} / n_{\mathrm{tot}} ! p(F) \mathrm{d} F,
\end{aligned}
$$

which defines a mixed-Poisson (MP) process. The study of such processes can be found mostly in the literature of statistical optics (Eq. (4) is often refereed to as Poisson-Mandel transform, Dainty 1975) and actuarial statistics (Grandel 1997). MP processes have gained much attention recently in the field of astronomy, especially for extrasolar planet detection by direct imaging, see e.g. Canales \& Cagigal (2001) and Aime \& Soummer (2004). In the latter works, the recorded flux is almost instantaneous (a few ms) and punctual (punctual pixels are assumed). In this case, the Poisson process is mixed by the statistical fluctuations of the speckles, which follow an exponential distribution. This leads to a Bose-Einstein distribution for the detected number of photons. In our case, the MP process is different.
Depending on the power of the scintillation noise, the mixing process is (at least for stars close to zenith) either Gaussian, lognormal or follows an $F$ distribution (Dravins et al. 1997a,b). An approximated expression can be found in the lognormal case in Diament \& Teich (1970). For stars far from the zenith, an analytical description is difficult because the perturbations of the wavefront are no longer weak (saturation regime).

To the author's knowledge, such a statistical description does not appear in the literature dealing with the analysis of photometric light curves. As we shall see in the following, this approach allows a precise description of how photon and scintillation noise are balanced for any given star's magnitude and observational parameters (through $\alpha$ and $\beta$ of Eqs. (2) and (3). The noise process is described here by the same model (4) irrespective of the star's magnitude. The two simple situations where either scintillation or photon noise dominates are two extreme approximations of this model.

As far as the overall number of noise photons is concerned, it is sufficient to evaluate the mean and the variance of the noise process (see Sect. 5 for more on these statistics). Irrespective of the particular distribution $p(F)$ of the mixing process, the mean of the MP process equals that of the Poisson process alone, and its variance is the sum of the variances of the two stochastic processes (photon noise and scintillation in our case) (Grandel 1997). Consequently, the average number of detected photons is $n_{\mathrm{sky}}+n_{*}$, and with Eq. (3) the associated variance is $n_{\text {sky }}+n_{n_{*}}+\beta^{2} n_{*}^{2}$. Hence, the overall (rms) noise, expressed in photons and including the joint effects of sky background, scintillation and photon noises becomes

$\Delta n_{\text {noise }}=\left(n_{\text {sky }}+n_{*}+\beta^{2} n_{*}^{2}\right)^{\frac{1}{2}}$.

Indeed, the above expression tends to $\Delta n_{s c}$ as the photon noise is negligible $\left(n_{\text {sky }}+n_{*} \ll \beta^{2} n_{*}^{2}\right)$, and to $\sqrt{n_{\text {sky }}+n_{*}}$ as the scintillation noise becomes negligible $\left(\beta^{2} n_{*}^{2} \ll n_{\text {sky }}+n_{*}\right)$.

The typical noise to be accounted for at Devasthal is presented for a 2 mmag amplitude signal in Fig. 1 (1 and $3 \mathrm{~m}$ telescopes). In all the figures, the stars are assumed at zenith. From Eq. (3), larger perturbations must be expected according to sec $Z$.

In Fig. 1, three regimes appear for the detection of oscillations: for $1 \mathrm{~m}$ class telescopes, scintillation dominates for stars brighter than $\approx 10$ th mag; scintillation and photon noises are of comparable influence up to $\approx 15$ th mag stars; photon noise and sky background dominate for fainter stars. In statistical terms, the doubly stochastic nature of the noise process is dominated by one of its stochastic components (scintillation) for bright stars. The noise process is clearly doubly stochastic for stars of intermediate magnitude, for which both noises prevail. For fainter stars, it is dominated by the effects of the other stochastic component (photon noise). In the intermediate magnitude region, the magnitude at which photon noise and scintillation are equal are indicated in the graphs.

Note that the sky background's influence is important for stars fainter than $\approx 15$ th mag (above which the overall noise deviates from the photon noise caused by the star only). These results are for dark nights: in the presence of moonlight, the influence of the sky background may affect the detection of variations in brighter stars.

\subsection{Remarks regarding the validity of the model}

We now consider the realism of this model because Eqs. (3) and (5) do not account for other atmospheric effects, in particular variations of the sky background, extinction and sky 

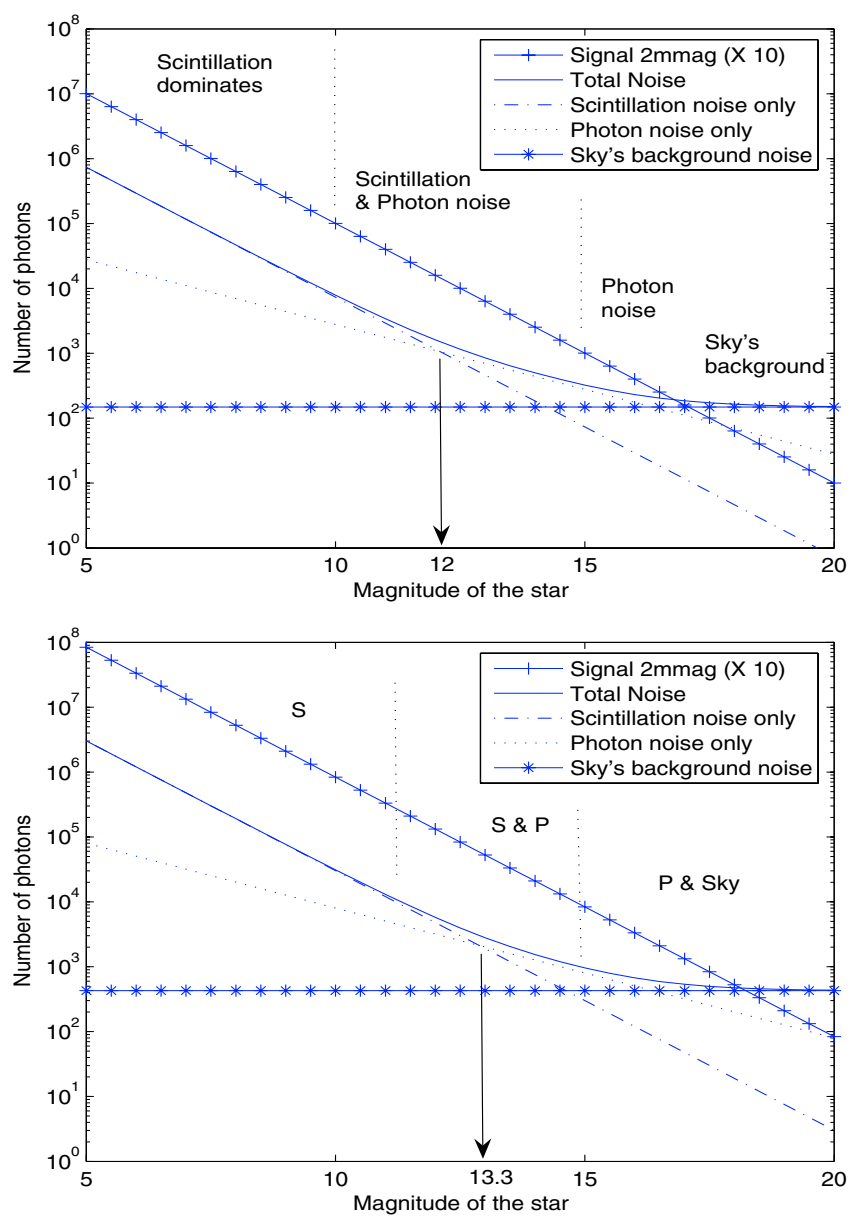

Fig. 1. Number of photons from signal and noise for a moonless night at the Devasthal site (the signal is magnified by 10 for visibility). Top: $1 \mathrm{~m}$. Bottom: $3 \mathrm{~m}$ telescope.

transparency. The first two noises can be efficiently removed from the raw data by carefully inspecting the light curve and using a channel dedicated to the sky. The third noise source, sky transparency variations, is more complicated to deal with. These variations take the form of random, non coherent oscillations with pseudoperiods of a few minutes to hours in the light curves. Such variations are attributed to variations in the atmosphere (viz. humidity, aerosols, dust; see Clarke 1980; Vid'machenko 1994; and Burnashev et al. 1991), whereas scintillation comes from fluctuations of the air's refractive index in the upper atmosphere. At low frequencies, it is not clear what the spectrum of scintillation actually looks like, nor what is the respective contribution of the two effects in the long time scale region (Dravins 1998). But what is clear is that variations in the chemical composition of the atmosphere/low frequency scintillation cause low frequency variations - whose power may be several times greater than the scintillation noise (see Fig. 2). Because these are incoherent oscillations, they tend to average out when one increases the observation duration - even if some cases are reported of exceptionaly coherent, short term sky transparency variations (during about 15 h, Kurtz 1984). The frequencies of these oscillations vary from night to night. It is well established however that in general, these perturbations occur at frequencies less than 1 or $2 \mathrm{mHz}$, above which the noise spectrum is approximately flat (see Fig. 2; and Sagar 1999; Martinez \& Kurtz 1994; Martinez et al. 2001; Joshi 2004; Sagar \& Mary 2005; Joshi et al. 2006).

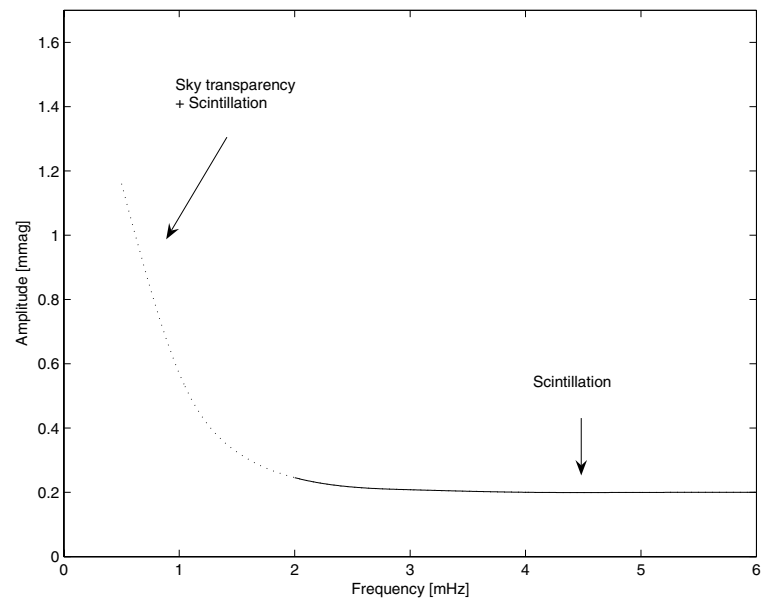

Fig. 2. A schematic representation of the noise peak level generated by atmospheric noise (both the sky transparency variations and scintillation) in the amplitude spectrum obtainable at Manora Peak, NainiTal with the 104-cm Sampurnanand telescope. Usually, the sky transparency variations have larger amplitude below 1.0 or $2 \mathrm{mHz}$. Their level drops to the scintillation noise level between 1 and $2 \mathrm{mHz}$, depending on the photometric quality of the night. On good nights, these sky transparency peaks are well separated from the frequencies of interest $(v \geq 1.0 \mathrm{mHz})$ indicating that we can detect the roAp oscillations above the scintillation noise level. Figure 1 indicates that the scintillation noise on a good photometric night can reach $\sim 0.2 \mathrm{mmag}$. This is below the characteristic amplitude variations of most known roAp stars (a few mmag typically), and comparable to the scintillation noise at the SAO site (see e.g. Martinez 1993; and Sect. 6).

In practice, the long term variations are first removed from the light curves when performing the data reduction (prewhitening, see Sect. 6). Indeed, this procedure is somewhat delicate in both cases of random and periodic long term stellar variations. For such stellar signals, the knowledge of what is noise and what is long term stellar variations may come from complementary observations of the same star, from some a priori information, or from the simultaneous observation of a comparison star - but most often, signal and noise cannot be distinguished ${ }^{1}$. In the prewhitened light curves however, the remaining noise is mainly caused by scintillation and photon noise on good nights. For rapid stellar variations, it is these noise sources, not sky transparency variations, that set their detectability. A detailed analysis of these aspects for some examples is given in Sect. 6 .

\section{Light curves}

\subsection{Signal}

In the light curve, the signal that we seek to detect is the stellar variation with respect to the average level of light (star plus sky background). Consequently, the apparent amplitude variation in the light curve $\Delta f_{\max , a}$ (with respect to star plus background), will be different from $\Delta f_{\max }$ (with respect to star only). We can show that they are related by

$\Delta f_{\text {max }, \mathrm{a}}=2.5 \log \left(1+\frac{\sqrt{2} \Delta n}{n_{*}+n_{\text {sky }}}\right) \approx \Delta f_{\text {max }}\left(1-\frac{n_{\text {sky }}}{n_{*}}\right)$.

1 This is the case in single-channel photometry, but even in twochannel photometry it is in general difficult to separate out drifts in the two channels from sky transparency variations, particularly at very low amplitudes and low frequency. 

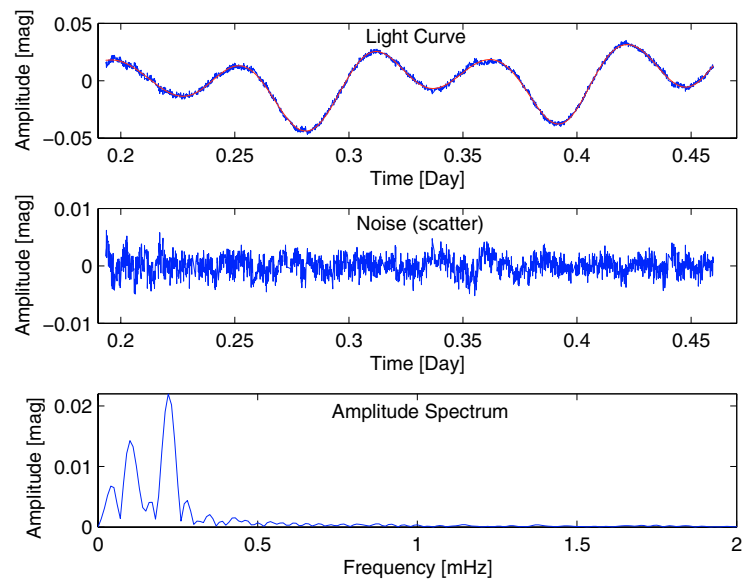

Fig. 3. Top panel: light curve of Am star HD 98851 ( $B$ mag $=7.72)$ obtained on HJD 2451594 at Manora Peak with the $104 \mathrm{~cm}$ telescope (Joshi 2003). The sky transparency variations are very low, and the signal's amplitude is quite large. The estimated scatter caused by the noise with respect to oscillation is plotted in the middle panel. The noise's rms level is about $1.6 \mathrm{mmag}$. Bottom panel: amplitude spectrum of the light curve. Two main frequencies $(0.21,0.10 \mathrm{mHz})$ are due to the star. The average noise level in the spectrum is $55 \mu \mathrm{mag}$. For a $7.4 \mathrm{~h}$ run, the present model (Eqs. (10) and (12)) yields $45 \mu$ mag. The maximum of the spectrum in the flat frequency region is $0.15 \mathrm{mmag}$. The level of the maximum from the model is $0.12 \mathrm{mmag}$ (Eq. (14)). In the light curve, $S N R_{t}=12$. In the spectrum, $S N R_{v}=400$ at $0.21 \mathrm{mHz}$ (cf. Eqs. (11) and (13)).

The fainter the stars, the less important the stellar variation relative to the background. For bright stars, $\Delta f_{\max , a}$ is equal to $\Delta f_{\max }$; this is not true for faint stars (see the decreasing curve in Fig. 9).

\subsection{Noise}

The effects of the noise are to add a stochastic component on a stellar signal. As shown in Fig. 3, the noise scatters the data points of the light curve.

Let us denote by $\Delta N$ the (rms) number of photons corresponding to this stochastic component ( $\Delta N$ can be caused by scintillation noise, photon noise or both). The average number of photons with respect to which the noise creates the scatter is $n_{*}+n_{\text {sky }}$. The (rms) variation in mag created by the scatter, $\Delta f_{\text {noise }}$, may be evaluated as

$\Delta f_{\text {noise }}=2.5 \log \left(1+\frac{\Delta N}{n_{*}+n_{\text {sky }}}\right)$.

With scintillation only, Eqs. (3) and (7) lead to a scatter of

$\Delta f_{\text {sc }}=2.5 \log \left(1+\frac{\beta n_{*}}{n_{*}+n_{\text {sky }}}\right)$.

With photon noise only, the scatter is

$\Delta f_{\mathrm{p}}=2.5 \log \left(1+\frac{1}{\sqrt{n_{*}+n_{\mathrm{sky}}}}\right)$,

and the scatter generated by both noises is

$\Delta f_{\text {noise }}=2.5 \log \left(1+\frac{\sqrt{n_{*}+n_{\mathrm{sky}}+\beta^{2} n_{*}^{2}}}{n_{*}+n_{\mathrm{sky}}}\right)$.

Again, it is readily checked that the above expression tends to Eq. (8) as photon noise is negligible, and to Eq. (9) as photon

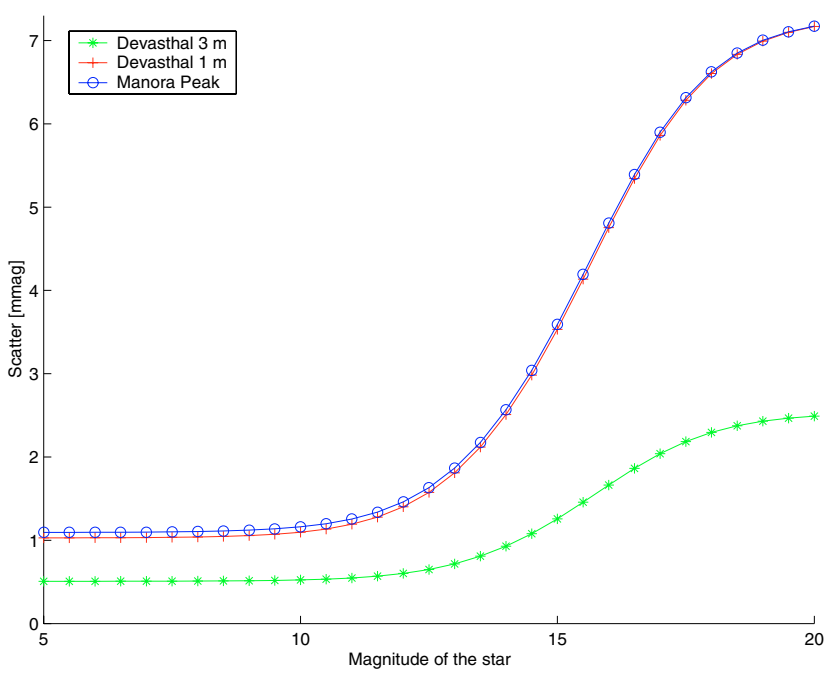

Fig. 4. Typical noise scatter (rms) in the light curves.

noise prevails. The overall scatter is plotted for the three observational settings in Fig. 4.

For bright stars, the scatter is mostly caused by scintillation and is constant in magnitude because the scintillation's power is proportional to the flux. The scatter increases with increasing photon noise, and finally converges to a constant value: for very faint stars, the sky background sets the average light level, and the corresponding random fluctuations set the scatter.

\subsection{SNR in the light curves}

We are interested in the light variation (with respect to the average light level) caused by the signal in comparison to the amplitude of the fluctuations caused by the noise. The higher the noise scatter, the less visible the signal. With Eqs. (2) and (5), the signal to noise ratio in the temporal domain $\left(S N R_{t}\right)$ can be defined as

$S N R_{t}=\frac{\Delta n}{\Delta n_{\text {noise }}}=\frac{\Delta n}{\left(n_{\text {sky }}+n_{*}+\beta^{2} n_{*}^{2}\right)^{\frac{1}{2}}}$.

Figure 5 plots the $S N R$ in the light curves for the three observational settings considered in this study, and for two different signals (20 and 2 mmag amplitude oscillations; the $\approx 20 \mathrm{mmag}$ signal is comparable to that of Fig. 3).

The transition regions mentioned in Fig. 1 set the shape of the $S N R$ curves. The $1 \mathrm{~m}$ telescope of Devasthal should present slightly better performance than its counterpart at Manora Peak because of higher altitude and lower extinction. For both $1 \mathrm{~m}$ telescopes and for stars brighter than 12 th mag, $\approx 2$ mmag corresponds to the lower amplitude limit below which the number of signal photons becomes inferior to that of noise photons $\left(S N R_{t}<1\right)$. As shown below, one can indeed detect much weaker signals if they are periodic, by analysing the Fourier spectrum of the light curves.

\section{Noise and SNR in the amplitude spectra}

The Fourier amplitude spectrum can be estimated via the Discrete Fourier Transform (DFT). Denoting by $T_{\text {tot }}$ the length of the run, the number of frequency bins in the bandwidth is $T_{\text {tot }} / T_{\text {int }}$, which corresponds also to the number of data points $N$. The expected value of the spectrum estimate in the 


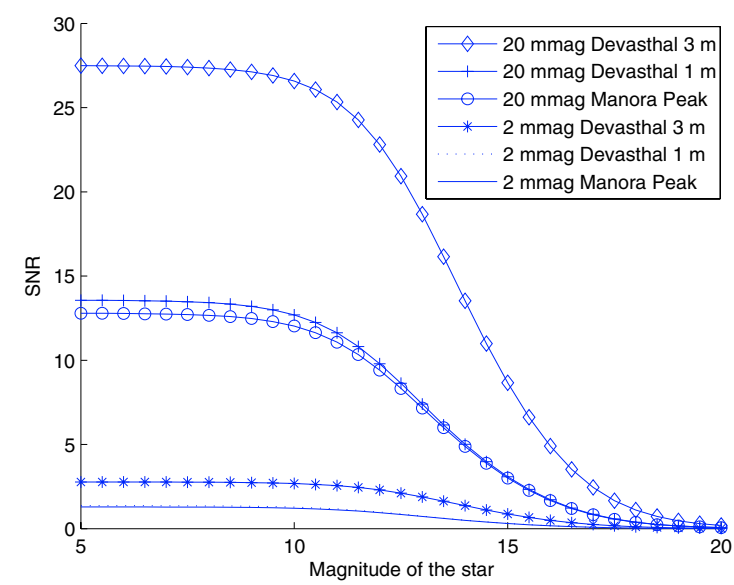

Fig. 5. Signal to noise ratio in the light curves vs. magnitude of the star when observing $\Delta f_{\max }=20 \mathrm{mmag}$ and $2 \mathrm{mmag}$ amplitude oscillations for the Manora 1-m telescope and the Devasthal 1-m and 3-m telescopes.

signal frequency bin is independent of $N$ with a proper normalisation and equals $\Delta_{\max , \mathrm{a}}$.

As for the noise, the expected level of the spectrum is approximately the same in all frequency bins because the scintillation noise's spectrum is approximately flat (cf. Fig. 2). By the Wiener-Khintchine theorem, the variance of the scatter in the light curve ( $\Delta f_{\text {noise }}^{2}$, Eq. (10)) corresponds to the sum of the contribution of the noise power spectral density in all the frequency bins. Consequently, the noise power $\Delta f_{\text {noise }}^{2}$ is spread over the whole bandwidth when going to the Fourier spectrum. The noise level is thus dependent on the number of frequency bins. The expected noise level can be evaluated as

$\Delta f_{\text {noise }, / \text { bin }} \approx 2 \frac{\Delta f_{\text {noise }}}{\sqrt{N}}$

The more data points, the less noise in each frequency bin. The $S N R$ at the signal's frequency $\left(S N R_{v}\right.$, defined as the ratio of the expected level of the signal's peak to the expected noise level in the amplitude spectrum) becomes simply (see for example Scargle 1982, Eq. (9))

$S N R_{v}=\frac{\Delta f_{\text {max }, \mathrm{a}}}{\Delta f_{\text {noise }, / \text { bin }}}=\sqrt{\frac{N}{2}} S N R_{t}$.

The expected noise level in the frequency bins is plotted in Fig. 6 for a $7 \mathrm{~h}$ run. Note that the contribution of scintillation to the total apparent noise vanishes for stars fainter than 14th mag because the corresponding power becomes negligible with respect to background fluctuations.

The $S N R_{v}$ (exp. (13)) obtained for a 2 mmag oscillation are compared in Fig. 7. These curves show that the SNR in the signal's frequency bin is fairly high $(\gg 1)$ for an oscillation of a few mmag. This means that on average, the peak of the signal will be $S N R_{v}$ times higher than the expected level of noise. In each bin, the amplitude spectrum is however a random quantity, whose standard deviation is close to the mean $\left(\Delta f_{\text {noise,/bin }}\right.$, Eq. (12), see e.g. Koopmans 1974, p. 265). Consequently, high peaks can be randomly generated by noise. When neither the frequency of the signal nor the $S N R$ are known a priori, it may be difficult to reliably detect the signal: many peaks may be signal candidates. Hence, the $S N R$ by itself is not enough: a confidence level corresponding to a given $S N R$ must be defined and evaluated.

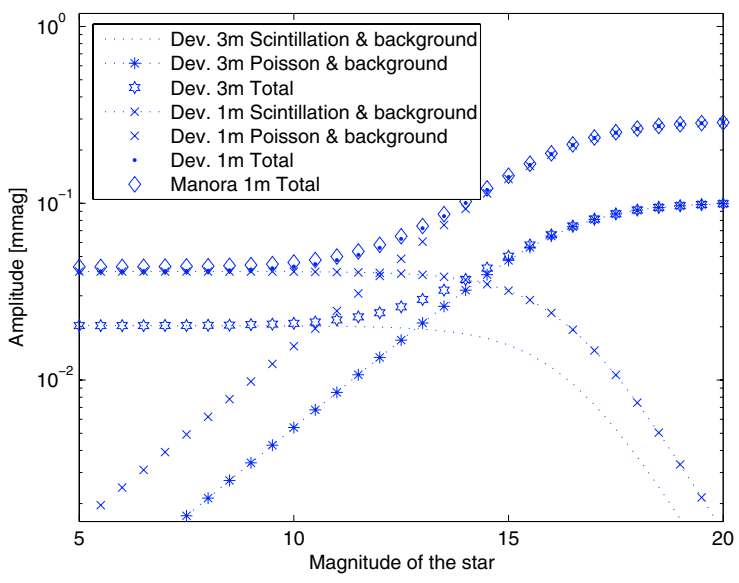

Fig. 6. Average noise level in the amplitude spectrum. For each observational setting, the contribution of scintillation (Eq. (8)), photon noise (Eq. (9)), and total noise contribution (Eqs. (10) and (12)) are displayed. The maxima are $\approx 3$ times higher. The run length is $7 \mathrm{~h}$. The average level and the maxima can be decreased by increasing the run length.

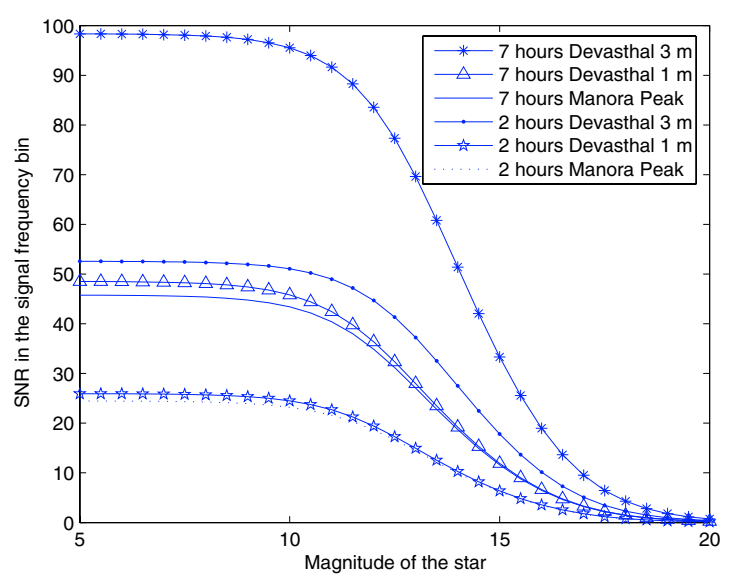

Fig. 7. Comparison of the signal to noise ratio in the amplitude spectrum when detecting a 2 mmag oscillation, for the three observational settings, and for 2 and $7 \mathrm{~h}$ runs.

\section{Confidence levels for the detection of periodic signals}

\subsection{Statistics of the periodogram with scintillation and photon noise}

In order to evaluate confidence levels for the detection of stellar oscillations, it is necessary to determine the statistics of the noise in the spectrum. It is customary for that purpose to assume that the noise is additive, white and Gaussian (e.g. Gilliland \& Brown 1992). This assumption seems questionable, since from Sect. 2 , the noise is MP in nature.

In the case of bright stars however (say, less than 11th mag), the contribution of the photon noise (Poisson component) is negligible (Figs. 1 and 6). For low scintillation levels, the scintillation is close to a lognormal distribution, and is best modelled by an $F$ distribution. Low scintillation means that $\beta$ in Eq. (3) is smaller than $\sqrt{0.2}=0.4$ (Dravins 1997a, p. 182). The lognormal probability density function is in turn close to a Gaussian distribution for very low scintillation level, i.e. for $\beta \ll 0.4$. In the light curves, a $\beta$ of 0.4 would correspond to a scatter 

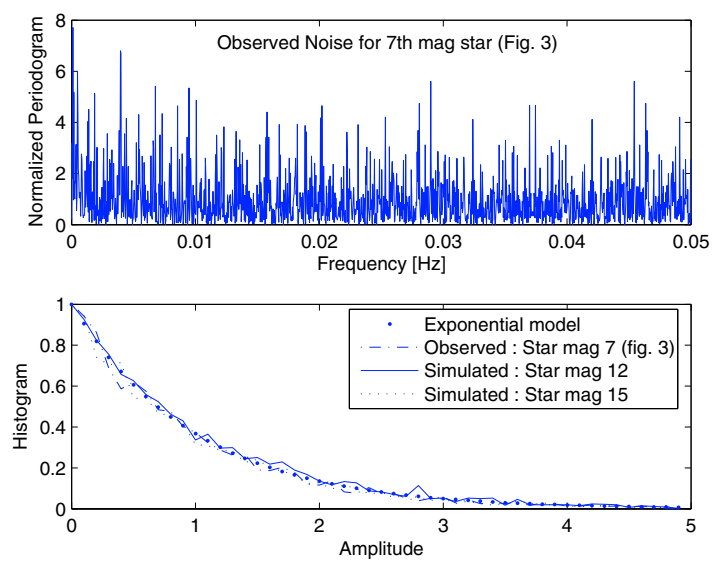

Fig. 8. Statistics of the periodogram. Top panel: normalised periodogram of the observed noise for a bright star: example of Fig. 3. Bottom panel: corresponding histogram of the periodogram, and histograms obtained for mixed-Poisson processes for 12th and 15th stars. The exponential model (corresponding to an additive white Gaussian noise) is accurate in all cases.

of $360 \mathrm{mmag}$. This is much more that the few mmag observed in practice in astronomical sties (see the example of Fig. 3, for which $\beta=0.002$ ). This means that for the telescopes considered here, the distribution of the scintillation is actually very close to Gaussian. The classical (assuming Gaussian noise) analysis of the periodogram statistics is clearly valid for bright stars. In this case, the periodogram $|F T(v)|^{2}$ is exponentially distributed (Scargle 1982). Figure 8 compares the empirical distribution of the periodogram of the noise corresponding to Fig. 3 to that of white Gaussian noise. The exponential distribution is clearly a good model.

For fainter stars, the noise process will be Poisson mixed by a Gaussian process, or purely Poisson for very faint stars. In both cases, it is not clear analytically what will be the distribution of the periodogram. To check this point we performed some numerical simulations. A mixed Poisson process was generated according to the observational parameters described in Sect. 2. The resulting histogram is plotted in Fig. 8, for stars of 12th mag (photon noise and scintillation are comparable) and 15 th mag (photon noise dominates the mixed-Poisson process). In all cases, the exponential model accurately reflects the distribution of the periodogram. We shall therefore use that model in the confidence analysis below, which follows directly from Scargle (1982).

\subsection{Confidence levels}

Since the signal's frequency is unknown, one should check how likely the noise is to randomly generate high peaks in the frequency bins. In order to do that, one can compare any given signal peak candidate to the statistics of the peaks' maxima. In the exponential model, the average level of the maxima in the amplitude spectrum is

$M \approx\left(\sum_{k=1}^{N / 2} \frac{1}{k}\right)^{\frac{1}{2}} \Delta f_{\text {noise } / \text { bin }}$

This level gives some insight into the statistical likelihood of high noise peaks. To illustrate this, let us consider the example of a 0.2 mmag amplitude oscillation (Fig. 9).

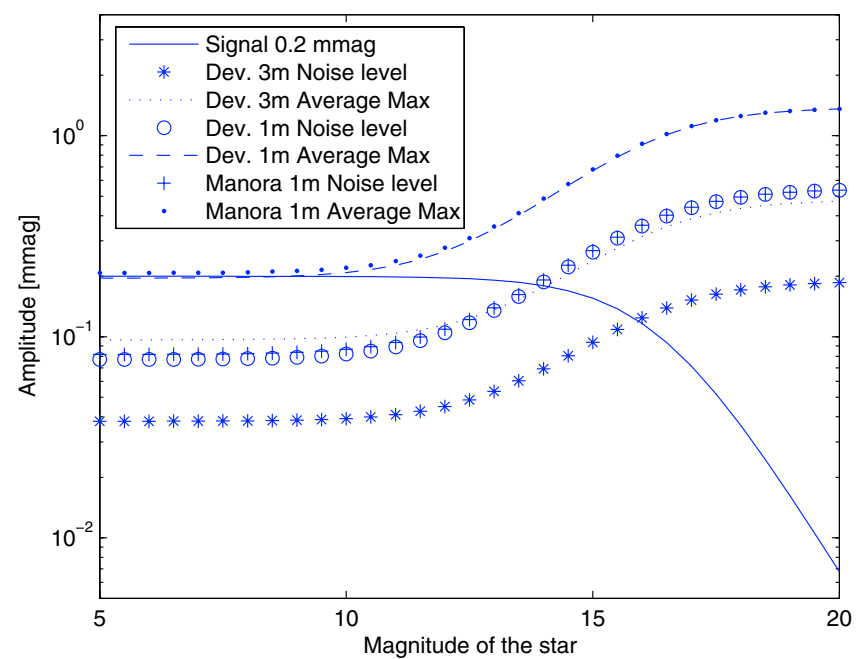

Fig. 9. Comparison of the apparent signal $\Delta f_{\text {max a }}$ (variation with respect to $F_{*}+F_{\text {sky }}$ ) to the average noise level ("Noise level" in the legend), and to the expected level of the maxima ("Average Max") in the amplitude spectrum obtained in $2 \mathrm{~h}$.

Firstly, the fainter the stars, the more difficult the detection of the stellar variation with respect to the background (full line; cf. Eq. (6)). Secondly, even if the average noise level is low with respect to the signal level, there may be many high peaks which make difficult the detection of the signal. In the case of the $1 \mathrm{~m}$ telescope at Manora Peak for example, the average noise level is about 0.08 mmag for bright stars observed for $2 \mathrm{~h}$ (crosses), but the average noise level of the maxima is about $0.2 \mathrm{mmag}$ (large dots), that is, as high as the signal (decreasing curve). The $S N R_{v}$ is more than 2 in this case, but peaks that are as high as the signal peak, though generated by noise, are statistically likely, preventing any reliable detection.

A useful quantity is therefore the confidence level corresponding to a given $S N R_{v}$. A confidence level of $C \%$ means that a peak which is $S N R_{v}$ times higher than the expected noise level will not have been generated by the noise $C \%$ of the time. $C$ is given by

$C \approx 100 \times\left(1-\mathrm{e}^{-S N R_{v}^{2}}\right)^{N / 2}$

The $S N R_{v}$ for this signal is compared for the three observational settings in Fig. 10 (top). The corresponding confidence levels (Eq. (15)) are plotted in the bottom figure. Even if the SNR is more than 1 in every case, the detection of a 0.2 mmag amplitude oscillation cannot be claimed at a significant confidence level (say, more than 95\%) with $1 \mathrm{~m}$ telescopes in $2 \mathrm{~h}$. With a $3 \mathrm{~m}$ telescope, a detection is possible at that level for stars brighter than magnitude 14.

An interesting question is that of the minimum amplitude variation $f_{\min }$ that can be detected with a given confidence level. With Eqs. (6) and (18) from Scargle (1982), we obtain

$f_{\text {min }}=2.5\left(1+\frac{n_{\text {sky }}}{n_{*}}\right) \log \left(1+\frac{2 \Delta n_{\text {noise }}\left(-\ln \left[1-C^{\frac{2}{N}}\right]^{1 / 2}\right.}{n_{*}+n_{\text {sky }}}\right)$.

This is plotted in Fig. 11 for the Manora Peak and Devasthal sites. In $2 \mathrm{~h}$, about $250-270 \mu \mathrm{mag}$ oscillations can be detected at a $99 \%$ confidence level with a $1 \mathrm{~m}$ telescope, for stars brighter than $\approx 11$ th mag. The minimum detectable amplitude increases to more than 1 mmag for 15 th magnitude stars. In the same duration, with a 3 m telescope at Devasthal, $\approx 120 \mu \mathrm{mag}$ 

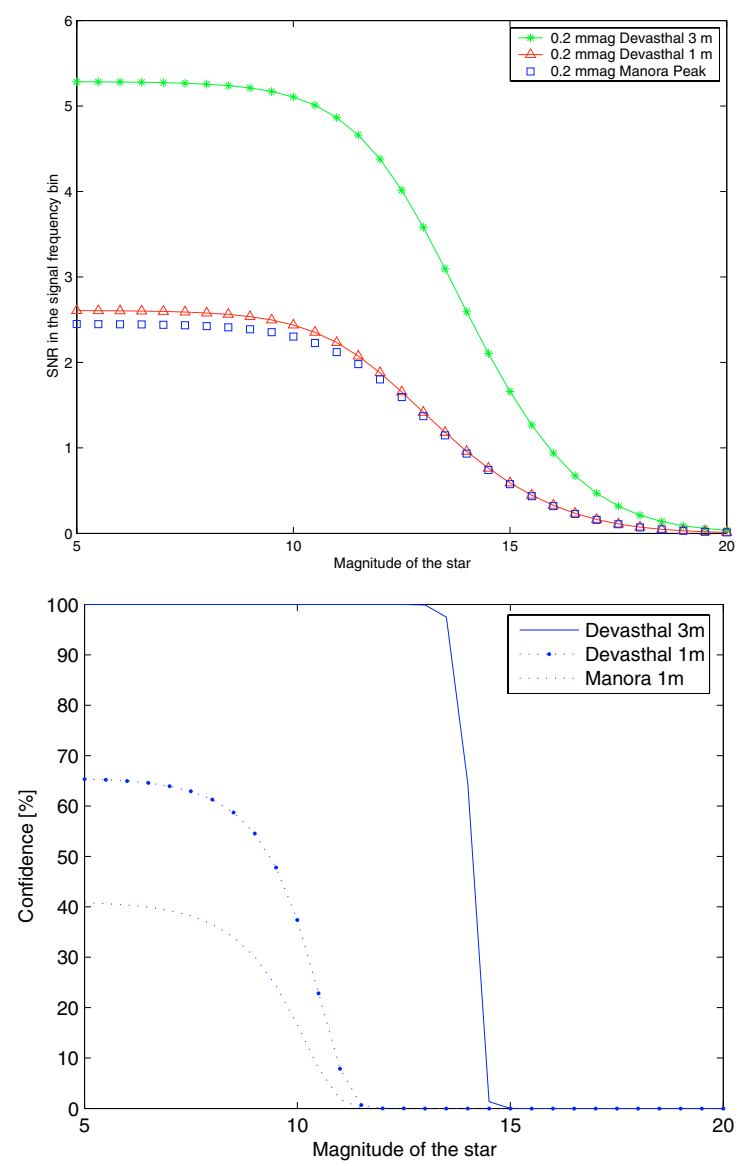

Fig. 10. Top: signal to noise ratio in the amplitude spectrum for a $0.2 \mathrm{mmag}$ amplitude oscillation (run of $2 \mathrm{~h}$, typical for roAp surveys). Bottom: corresponding confidence level.

variations would be detected at a 99\% confidence level up to 12 th magnitude stars. At $f_{*}=15, \approx 400 \mu$ mag oscillations would still be detected. In a $7 \mathrm{~h}$ run and for bright stars, $f_{\min }$ falls to $\approx 140-150 \mu \mathrm{mag}$ for the $1 \mathrm{~m}$ telescopes, and to $70 \mu \mathrm{mag}$ with a $3 \mathrm{~m}$ telescope at Devasthal. For bright stars, the gain in the minimum amplitude that can be detected with a $3 \mathrm{~m}$ telescope is thus a factor 2 over $1 \mathrm{~m}$ telescopes. As can be noticed from the curves of Fig. 11, this gain increases for fainter stars (factor 3 for $f_{*}=15$ ). The advantage of a large telescope is thus twofold: firstly, it allows one to detect smaller oscillations in bright stars (lower scintillation noise); secondly, with increasing star's magnitude, the lower detection limit increases relatively more slowy than for smaller telescopes (taking advantage of both lower scintillation and higher flux).

\section{Further comparisons with real data and discussion}

\subsection{Data acquisition and reduction}

For the NainiTal-Cape Survey, high-speed photometric observations of Ap and Am star candidates are carried out from ARIES (Manora Peak, NainiTal) using a three-channel fast photometer attached to the ARIES 104-cm telescope (Sagar 1999; Ashoka et al. 2000). The time-series photometric observations consist of continuous 10-s integrations obtained through a Johnson $B$ filter. This filter is expected for roAp stars to yield the highest amplitude variations and to maximize the number of counts.

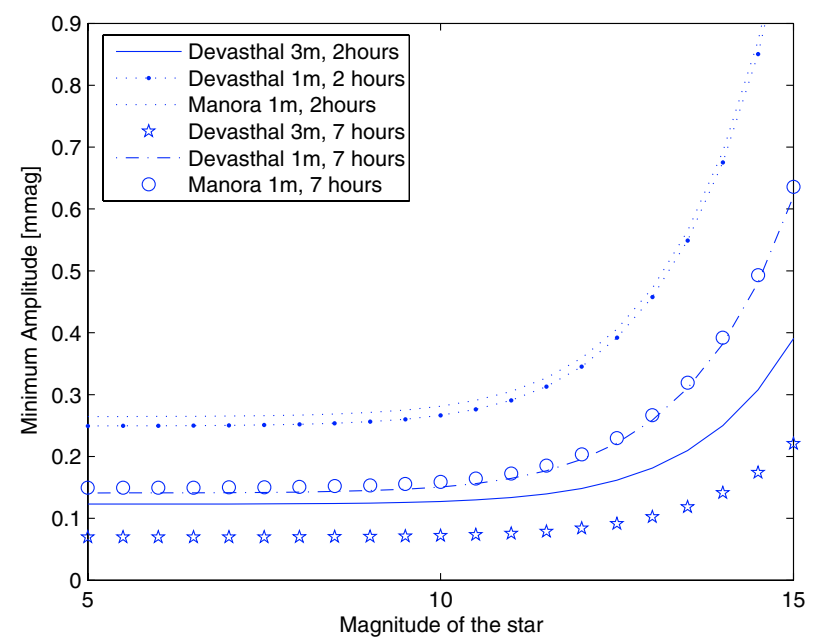

Fig. 11. Minimum amplitude that can be detected with $99 \%$ confidence for the three observational settings considered in this paper.

An aperture of $30^{\prime \prime}$ is used to minimize flux variations caused by seeing fluctuations and guiding. The observing protocol is simple. We acquire time-series photometric observations of the candidate stars for 1 to $3 \mathrm{~h}$ in order to be able to reveal both roAp and $\delta$ Scuti pulsations. The data reduction process comprises the following steps: (a) visual inspection of the light curve to identify and remove all obvious bad data points; (b) correction for coincident counting losses; (c) measurements of sky brightness produced by interpolating the points with a piecewise linear function; and (d) correction for the atmospheric extinction $(x(\lambda)=0.26$ for Manora Peak). After applying these corrections, the time of the mid-points of the observations are converted into heliocentric Julian dates (HJD) with an accuracy of $10^{-5}$ day $(\approx 1 \mathrm{~s})$. The reduced data comprise a time-series of the HJDs (days) and amplitude of variations (mag) with respect to the mean of the run. These data are analyzed using an algorithm based on the Discrete Fourier Transform (DFT) for unequally spaced data (Deeming 1975; Kurtz 1985). The DFT of the time-series produces an amplitude spectrum of the light curve. Long term oscillations that are likely to be produced by sky transparency variations are then removed from the light curve (prewhitening). The knowledge of what is noise and what is long term stellar oscillation may come from complementary observations of the same star, or from the simultaneous observation of a comparison star. This procedure is delicate, and makes the determination of $\delta$-Scuti stars difficult unless they have large amplitudes. The problem is less accute for roAp stars whose pulsations are found at higher frequencies.

\subsection{Comparison of the model with real data}

The examples below are drawn from the null results of the NainiTal-Cape Survey (see Joshi et al. 2006). We first focus on the scatter caused by scintillation in the light curves. The model above leads for bright stars (less than 11th mag) and for $1 \mathrm{~m}$ telescopes to a typical scatter in the light curves of $\approx 1.1 \mathrm{mmag}$. For such stars, the typical scatter actually observed during the best nights at Manora Peak is between 1 and 2 mmag. The example shown in Fig. 3, with a scatter of $1.6 \mathrm{mmag}$, is representative of a good night in NainiTal. This level is comparable to that observed at SAO, Cape Town, South Africa (Martinez 1993) and is typical 
Table 1. Some stars observed during the NainiTal - Cape Survey (null results from Joshi et al. 2006).

\begin{tabular}{lccccccc}
\hline \hline Name & $\alpha_{2000}$ & $\delta_{2000}$ & $V_{\mathrm{m}}$ & Spectral type & $\begin{array}{c}\text { Time } \\
(\mathrm{h})\end{array}$ & $\begin{array}{c}\text { Max: observed } \\
(\mathrm{mmag})\end{array}$ & $\begin{array}{c}\text { Max: model } \\
(\mathrm{mmag})\end{array}$ \\
\hline HD 154 & 000624 & +343624 & 9.27 & F0 & 1.54 & 0.50 & 0.26 \\
HD 416 & 000851 & +371256 & 9.19 & A5 & 1.49 & 0.59 & 0.26 \\
HD 1607 & 002027 & +222847 & 9.06 & F0 & 1.70 & 0.24 & 0.24 \\
HD 37009 & 053812 & +503158 & 8.41 & F0 & 2.25 & 0.32 & 0.21 \\
HD 41786 & 060802 & +211743 & 7.64 & F0 & 1.37 & 0.43 & 0.25 \\
HD 47311 & 064001 & +423356 & 9.01 & F0 & 1.85 & 0.28 & 0.23 \\
HD 57955 & 072348 & +065646 & 8.00 & A2 & 1.08 & 0.26 & 0.28 \\
HD 73174 & 083737 & +194358 & 7.95 & Am & 0.72 & 0.14 & 0.33 \\
HD 76310 & 085559 & +214133 & 8.82 & Am & 2.00 & 0.26 & 0.22 \\
HD 81722 & 093349 & +774134 & 9.11 & G5 & 0.76 & 0.31 & 0.34 \\
HD 85216 & 095053 & +191925 & 8.68 & A3 & 0.71 & 0.21 & 0.34 \\
HD 90011 & 102327 & +021513 & 9.06 & F0 & 1.41 & 0.32 & 0.26 \\
HD 115722 & 131825 & +355714 & 9.84 & Am & 1.09 & 0.29 & 0.33 \\
HD 144999 & 160738 & +285943 & 8.1 & A3 & 2.04 & 0.78 & 0.21 \\
HD 168605 & 181950 & +191017 & 8.22 & A0p & 1.66 & 0.41 & 0.23 \\
HD 223247 & 234746 & +282426 & 8.43 & F0 & 1.18 & 0.34 & 0.27 \\
\hline
\end{tabular}

of other good photometric sites such as La Silla, Canary Islands, Granada and Sutherland (see the survey of Fossat 1984).

It is not rare however that the observed scatter is superior to 1 or 2 mmag. This may arise for several reasons. Firstly, sky transparency variations contribute to the overall scatter (this is discussed further below). In the raw data, their contribution can be large. These variations are however removed by prewhitening, so that their final contribution is small for good nights. Secondly, the stars are not always at zenith, and scintillation is expected to be stronger in this case. Thirdly, Eq. (3) is an empirical model; the power of scintillation indeed varies from night to night according to atmospheric conditions. Another reason may be the particular latitude of the sites considered here $\left(29^{\circ} 27^{\prime}\right)$ : the wind speeds are known to be maximum at $\approx 30^{\circ}$ latitudes (jet streams), and to influence the scintillation power (Dravins 1998). By using the general Eq. (3), which does not account for the site's latitude, one might consequently underestimate the average contribution of the scintillation for these latitudes. Other reasons can be the increased light pollution towards the city of NainiTal, forest fires occuring from April to June, as well as smog and dust.

For the $3 \mathrm{~m}$ telescope at Devasthal, Fig. 4 shows that the scintillation would cause an almost $500 \mu$ mag scatter for bright stars. For comparison, Brown \& Gilliland (1994) reported observations of the scatter at Kitt Peak and Mauna Kea of about $200 \mu \mathrm{mag}$ for $4 \mathrm{~m}$ telescopes, $T_{\text {int }}=60 \mathrm{~s}$. For the corresponding observational setting, the present model yields $180 \mu \mathrm{mag}$.

We shall now turn to the noise in the amplitude spectrum as observed for stars of the NainiTal-Cape survey in which no pulsations could be detected (null results). The eight columns of Table 1 list respectively, for 16 stars, the HD number, right ascension $\alpha_{2000}$, declination $\delta_{2000}$, visual magnitude $V_{\mathrm{m}}$, spectral type, total duration of the observation in hours, the average level of the peaks above $2 \mathrm{mHz}$ for each run, and the corresponding level obtained from the model. For this last column, we used in Eq. (2) the values 0.85 for the reflectivity of the primary and secondary mirrors, 0.9 for the Fabry Lens used with our photometer, and 0.3 for the quantum efficiency (Sagar \& Mary 2005). As mentioned in the Introduction, the detector noise is neglected to establish the noise for these bright stars. The spectra corresponding to the stars of Table 1 are shown in Fig. 14, along with the results of the model.
These spectra show firstly that the quality of the observation varies from night to night. Secondly, the tallest peak is almost never found in the $[0-0.5] \mathrm{mHz}$ frequency region, because prewhitening has removed the largest contribution of the sky transparency variations. Thirdly, the average level of the peaks is often higher in the low frequency region (below 1,2 or sometimes $3 \mathrm{mHz}$ ) than above, and decreases towards higher frequencies. This higher level is mainly caused by residual sky transparency variations. Above the low frequency region, the spectrum flattens to a level which corresponds to the scintillation noise. This right away tells us that the detection of an oscillation in the low frequency region is more difficult than at higher frequencies because the noise level is higher. Hence, detecting $\delta$ Scuti-like pulsations (longer than about $0.5 \mathrm{~h}$ ) is more difficult - unless they have very large amplitude, as in the example of Fig. 3. This observational fact might explain why long period pulsations are actually not detected in evolved roAp stars, although their existence is predicted theoretically (see the discussion of Cunha 2002).

We shall now characterize more precisely how sky transparency variations affect the detection. The frequency where the tallest peak occurs gives an idea of the frequency region where these variations are active, and its amplitude gives the power of these fluctuations. This tallest peak can therefore be considered as the detection limit in the low frequency region. For each prewhitened light curve of 110 stars from the null results, we selected the tallest peak; the distribution of these detection limits is displayed in Fig. 12.

In most cases, the tallest peak has an amplitude in the range [0.5-1.5] mmag. For this distribution, the mean and median values are respectively 1.52 and $1.25 \mathrm{mmag}$. $1.5 \mathrm{mmag}$ is less than the largest amplitude of many known roAp stars, meaning that this sensitivity level is in good agreement with the purpose of such a survey. On the other hand, the number of roAp stars having small amplitude pulsations is expected to increase dramatically below $1.5 \mathrm{mmag}$, so that efforts towards pushing down this limit would most probably lead to the discovery of many new variables.

If we now turn to the frequency at which the tallest peak occurs, we obtain the distribution of Fig. 13.

Again it can be observed that the prewhitening procedure has removed the peaks at very low frequency. Usually, the highest peak is found in the low frequency region of 0.5 to $2 \mathrm{mHz}$; 


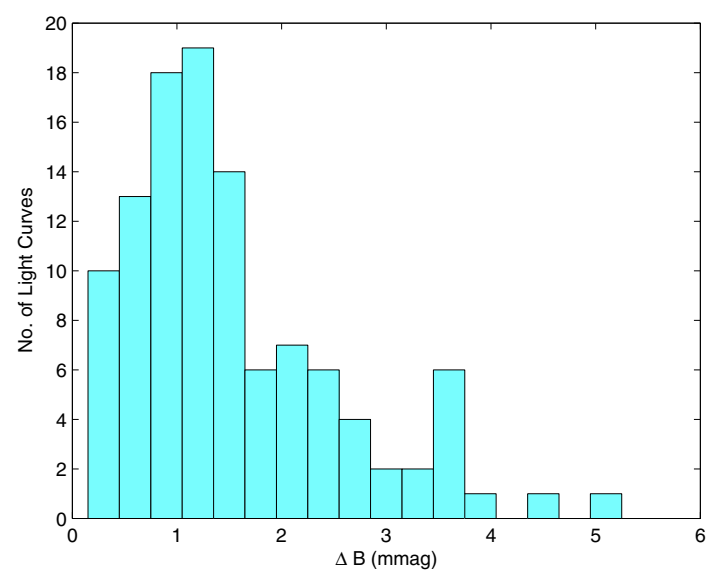

Fig. 12. Distribution of the detection limits (as the tallest peak in the spectrum) in the NainiTal-Cape Survey.

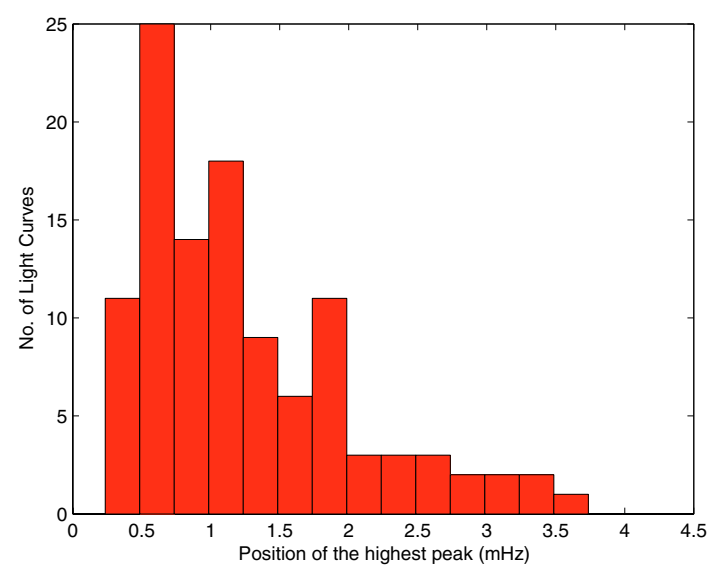

Fig. 13. Distribution of the positions of the tallest peaks in the prewhitened light curves.

the median value is $1.04 \mathrm{mHz}$ and the mean $1.25 \mathrm{mHz}$. The occurrence of the tallest of these frequencies is due to residual sky transparency variations, but probably also to undetected $\delta$ Scuti and roAp stars. Above about $2 \mathrm{mHz}$, the distribution tends to flatten, meaning that sky transparency variations are relatively much less active in this region. It is not clear whether the bump around $1.9 \mathrm{mHz}$ is caused by sample noise or by a real spurious effect (instrumental noise like a telescope drive error for example).

With these examples in mind, one sees that assessing a criterion to derive a detection limit, with the purpose that this limit is both general and significant can be difficult. The reasons are that the level of the sky transparency variation, and the frequency region where the level of sky transparency variations reaches scintillation noise level varies from night to night. For those nights where sky transparency variations affect only the low frequency regions however, it is scintillation noise (allied to photon noise for fainter stars) that sets the actual detection limit for the stellar pulsations. This can be seen from the spectra of Fig. 14. The corresponding scintillation noise levels are compared numerically in the last two columns of Table 1. For good nights, the MP model (dashed line) evaluates fairly accurately the noise level in the flat frequency region (stars HD 1607, HD 47311, HD 57955, HD 76310, HD 81722, HS 115722). This shows that for good atmospheric conditions, the model provides a useful evaluation of the achievable detection limits of the astronomical site. On exceptional nights, the observations are better than the model (HD 73174). On other nights, they are slightly worse (HD 90011, HD 223247), or much worse on non photometric nights (HD 154, HD 416, HD 144999).

These results are very similar to those obtained at the SAO site and discussed by Martinez \& Kurtz (1994) (see in particular their Figs. 1 and 2 for a comparison of Figs. 12 and 13). From their observational experience at SAO, these authors report a detection limit of $0.35 \mathrm{mmag}$ in $1 \mathrm{~h}$, for bright stars and with a $1 \mathrm{~m}$ telescope (p. 134). For the corresponding parameters and for a 7 mag star, the present model yields a detection limit of 0.36 mmag with $99 \%$ confidence.

\section{Summary and conclusions}

This work has attempted a precise evaluation of the statistical detection performances of stellar variability using ground based fast photometry. This evaluation was mainly illustrated by the studies of two astronomical sites, Devasthal and Manora Peak (1 and $3 \mathrm{~m}$ telescopes), but comparison with other sites was provided as well. We neglected sky transparency variations (low above 1 or $2 \mathrm{mHz}$ ), the noise caused by the detector was not accounted for, and the stars were assumed at zenith. The results presented here are thus best possible detection performances.

The overall noise in the light curve follows a MP process (photon noise mixed by scintillation). These statistics and results from atmospheric physics allowed us to quantify the respective contribution of the noises. The MP nature of the noise (Gaussian, Poisson mixed with Gaussian, Poisson) changes according to the magnitude of the variable star and to the astronomical site (Sects. 2 and 5). Consequently, 3 regimes appear for the detection (scintillation; scintillation and photon noise; photon noise and sky background). Such a statistical description does not seem to appear in the literature of photometric time series analysis. It however allows a precise understanding of how photon and scintillation noise are balanced for any given site and observational parameters. The noise process is described by the same model (Eq. (4)) irrespective of the star's magnitude. The two simple situations where either scintillation or photon noise dominates are two extreme approximations of this model.

For the Indian sites, the magnitudes marking the frontiers of the three regimes are about 0 mag and $15 \mathrm{mag}$. The proposed model in terms of scatter in the light curves (Sect. 3), noise in the amplitude spectrum and SNR (Sect. 4) matches observations made at Manora Peak, and is in good agreement with observations reported for other sites.

As far as the statistics of the amplitude spectrum is concerned, the exponential model was shown to be accurate whatever the brightness of the star (Fig. 8). It follows from this model that for stars brighter than $11 \mathrm{mag}$, about 250 to $270 \mu \mathrm{mag}$ oscillations can be detected at a $99 \%$ confidence level with $1 \mathrm{~m}$ telescopes in $2 \mathrm{~h}$. This increases to more than $1 \mathrm{mmag}$ for 15 th magnitude stars. With a $3 \mathrm{~m}$ telescope at $2500 \mathrm{~m}$, $120 \mu$ mag variations could be detected at a $99 \%$ confidence level in $2 \mathrm{~h}$ up to 12 th magnitude stars. At $f_{*}=15$, about $400 \mu \mathrm{mag}$ oscillations could still be detected. A larger telescope is advantageous with respect to smaller ones for two reasons. The first one is that it allows the detection lower amplitude pulsations. The second reason is less obvious: the comparatively higher gain in the amplitude detection is increased when the stars become fainter (Fig. 11). Indeed, a gain of a factor of 3 in the lower amplitude detection limit may not justify the time and cost of using a 3 times larger telescope. On the other hand, in the particular case of roAp stars, most surveys use typically $1 \mathrm{~m}$ telescopes 

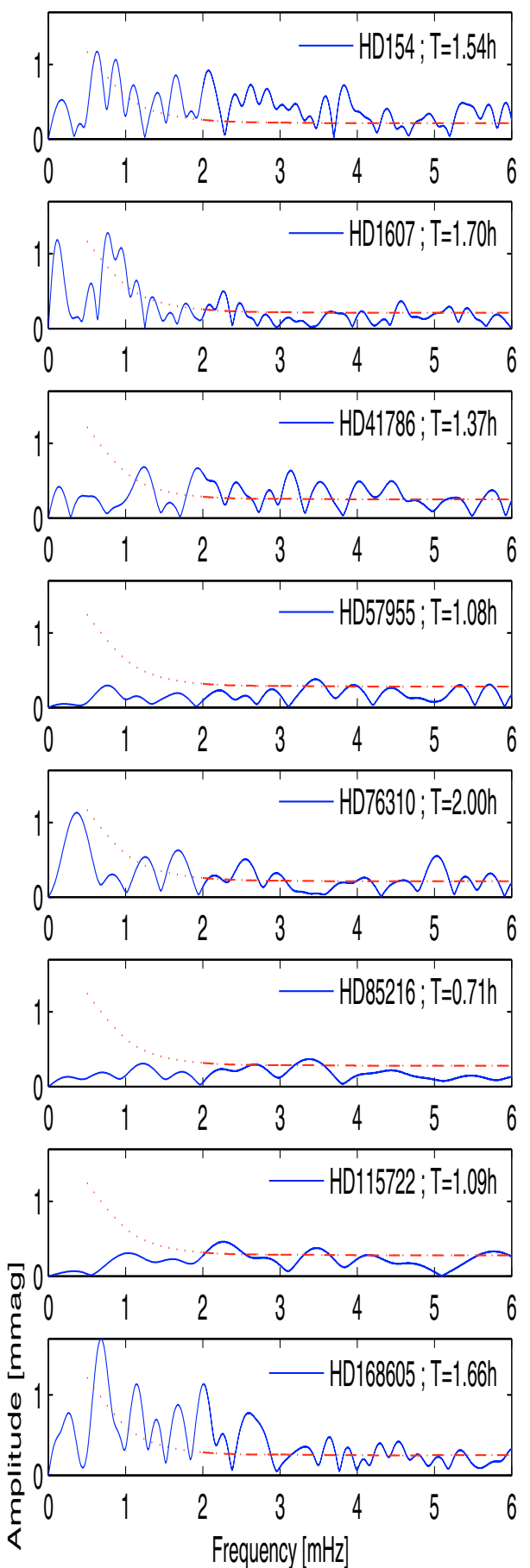
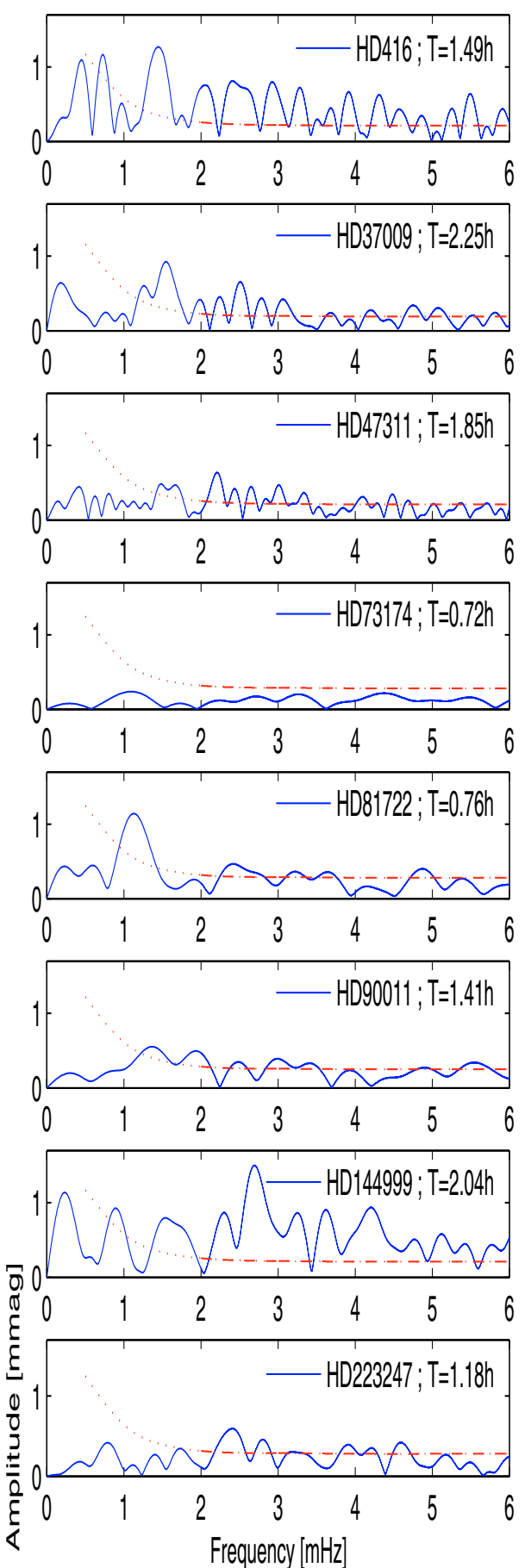

Fig. 14. Examples of spectra obtained at Manora Peak for the NainiTal-Cape Survey. The dashed line indicates the level of the peaks maxima according to the model, for the observation duration mentioned in the insets and the star's magnitude (Table 1). The dotted line is a schematic representation of the low frequency tail based on the null results from the Survey.

or less (Martinez 1993; Handler \& Paunzen 1999; Dorokhova \& Dorokhova 1998). So very few objects above 11 th magnitude have consequently been observed in such variability surveys, and one may argue that this is a large number of variable candidates to be observed with large telescopes. Although true, this view should be moderated because for large ( $3 \mathrm{~m}$ or more) telescopes, spectroscopic observations can be used to detect new variables (see the review of Kurtz 2005; and Elkin et al. 2005, for the recent discovery of the longest period in a roAp star using spectroscopy).
Acknowledgements. The author wishes to thank Brijesh Kumar, Santosh Joshi and Manoj K. Srivastava for several useful discussions, as well as the anonymous referee for valuable comments.

\section{References}

Aime, C., \& Soummer, R. 2004, ApJ, 612, 85

Ashoka, B. N., Seetha, S., Raj, E., et al. 2000, BASI, 28, 251

Breger, M. 2000, in Delta-Scuti and Related Stars, ed. M. Breger, \& H. Montgomery (San Francisco: ASP), ASP Conf. Ser., 210, 3 Brown, T. M., \& Gilliland, R. L. 1994, ARA\&A, 32, 69 
Burnashev, V. I., Burnasheva, B. A., Merkulova, B. I., \& Metik, L. P. 1991, Bull. Crimean Astrophys. Obs. 83, 140 (Izv. Krymskoi Astrofiz Obs. 83, 156)

Canales, V. F., \& Cagigal, M. P. 2001, Opt. Lett., 36, 737

Christensen-Dalsgaard, J. 2003, Lectures Notes on Stellar Oscillations, available at www .asteroseismology.org

Clarke, D. 1980, MNRAS, 190, 641

Cunha, M. S. 2002, MNRAS, 333, 47

Deeming, T. J. 1975, Ap\&SS, 36,137

Dainty, J. C. 1975, Laser Speckle (Berlin, Heidelberg, New York: Springer Verlag)

Diament, P., \& Teich, M. C. 1970, J. Opt. Soc. Am., 60, 1489

Dorokhova, T. N., \& Dorokhov, N. I. 1998, in Proc. of the 26th meeting of the European Working Group on CP stars, ed. P. North, A. Schnell, \& J. Ziznovský, Contributions of the Astronomical Observatory Skalnaté Pleso, Slovak Academy of Sciences, 338

Dravins, D., Lindegren, L., Mezey, E., \& Young, A. T. 1997a, PASP, 109, 173

Dravins, D., Lindegren, L., Mezey, E., \& Young, A. T. 1997b, PASP, 109, 725

Dravins, D., Lindegren, L., Mezey, E., \& Young, A. T. 1998, PASP, 110, 610; erratum PASP, 110, 1118 (1998)

Elkin, V. G., Riley, J. D., Cunha, M. S., Kurtz, D. W., \& Mathys, G. 2005, MNRAS, 358, 665

Fossat, E. 1984, Proc. of the Workshop on Improvements to Photometry (NASA Conf. Pub.), 2350, 68

Gilliland, R. L., \& Brown, T. M. 1992, Pub. of ASP, 104, 582

Girish, V. 2004, Ph.D. Thesis, Indian Institute of Science, India

Grandell, J. 1997, Mixed Poisson Processes, Monographs on Statistics and Applied Probability (Chapman \& Hall)

Handler, G., \& Paunzen, E. 1999, Ap\&SS, 135, 57

Joshi, S. 2003, MNRAS, 344, 431

Joshi, S. 2004, Ph.D. Thesis, Kumaon University, NainiTal, India
Joshi, S., et al. 2006, A\&A, submitted

Kholopov, P. N., et al. 1998, The combined Table of General Catalogue of Variable Stars, Vol. I-III (Moscow: Sternberg Astron. Inst.), improved electronic version Vol. I-V, available via anonymous ftp to ftp://ftp.sai.msu.su/pub/groups/cluster/gcvs/gcvs/iii/

Koopmans, L. H. 1974, The Spectral Analysis of Time Series (New York and London: Academic Press)

Kumar, B., Sagar, R., Rautela, B. S., et al. 2000, BASI, 28, 675

Kurtz, D. W. 1984, in Proc. of the Workshop on Improvements to Photometry (NASA Conf. Publ.), 2350, 56

Kurtz, D. W. 1985, MNRAS, 213, 773

Kurtz, D. W. 2005, JAA, 26, 123

Martinez, P. 1993, The Cape Rapidly Oscillating Ap Star Survey, Ph.D. Th., Univ. of Cape Town

Martinez, P., \& Kurtz, D. W. 1994, MNRAS, 271, 129

Martinez, P., Kurtz, D. W., Ashoka, B. N., et al. 2001, A\&A, 371, 1048

Mary, D. L. 2005, JAA, 26, 283

Mayya, Y. D. 1991, JAA, 12, 319

Nather, R., Winget, D., Clemens, J., Hansen, C., \& Hine, B. 1990, ApJ, 361, 309

Poretti, E. 2002, in Observational Aspects of Pulsating B and A Stars, ed. C. Sterken, \& D. W. Kurtz, ASP Conf. Ser., 256, 269

Reed, M. D., Kilkenny, D., Kawaler, S. D., et al. 2000, BaltA, 9, 183

Sagar, R. 1999, Current Sci., 77, 5, 643

Sagar, R., Stalin, C. S., Pandey, A. K., et al. 2000, A\&AS, 144, 349

Sagar, R., \& Mary, D. L. 2005, JAA, 26, 339

Scargle, J. D. 1982, ApJ, 263, 835

Vid'machenko, A. P. 1994, Kin. Phys. Celestial Bodies 10, No. 5, 52 (Kin. Fiz. Nebesnykh Tel 10, No. 5, 62)

Young, A. T. 1967, AJ, 72, 747 